\title{
Effect of Inertia Level on Aerodynamic Derivatives of a wedge in Hypersonic Flow
}

\author{
Pavitra S. ${ }^{1}$, Lavanya S. ${ }^{2}$, S. A. Khan ${ }^{3}$ \\ ${ }^{I}$ Assistant Professor, Department of Mathematics, B. I. T, Mangalore, Karnataka, India. \\ ${ }^{2}$ Professor, Department of Mathematics, P.A.C.E, Mangalore, Karnataka, India. \\ ${ }^{3}$ Professor, Department of Mechanical Engineering, Faculty of Engineering, IIUM, Gombak Campus, Kuala \\ Lumpur,Malaysia.
}

\begin{abstract}
In this paper a 2-dimensional slender bodies in hypersonic flow at high angle of attack with different Mach number has been implemented. By utilizing the concept of a piston moving in a cylinder at any speed, relations for stability derivatives are obtained for a wedge, which depends on the flight Mach number and semi vertex angle of the wedge. Stiffness derivatives decreases linearly with the increase in the Mach number for $h=$ 0, 0.2, 0.4 but for Mach number 10 and above the steady state is achieved and stability derivative becomes independent of Mach number. For pivot positions $h=0.6,0.8$, and 1.0 unlike for $h=0$ to 0.4 the stability derivative increases up to Mach 10 and again it becomes independent of Mach number. The variation of the damping derivatives is unaltered for all the values of the pivot positions. The damping derivative decreases marginally and then it becomes independent of Mach number. Viscous effects, secondary wave reflections are neglected in the present study. In the present study the real gas effects and leading edge bluntness have not been taken into considerations.
\end{abstract}

Keywords - Angle of attack, Mach number, Stability derivatives.

\section{Introduction}

The piston theory concept was used by Hayes ${ }^{[1,2]}$ and Light hill ${ }^{[4]}$ for finding the unsteady flow near thin airfoils for hyper sonic flow. Light hill ${ }^{[4]}$ considered the pitching oscillation and co-related the unsteady problem to that of flow of a gas inside a tube driven by a piston. Ghosh \& Mistry ${ }^{[5]}$ have given a theory for the quasi steady flow over the oscillating 2-D wedge which has been further extended by Ghosh ${ }^{[5,6]}$ to solve the axi symmetric flow problem. Ghosh ${ }^{[5,6]}$ applied piston theory for non-planar steady and unsteady cases.

The stability derivatives have been obtained for sharp wedges formed by two flat plates. Crasta \&Khan ${ }^{[7-10]}$ have given a theory for the quasi steady flow for the wedge which is an extension of Ghosh's theory, which predicts the stability derivatives in pitch and roll. A similitude implemented for hypersonic flow with planar wedge by attaching bow shock at high incidence. Sychev ${ }^{[3]}$ developed a theory in hypersonic flow for 3-D bodies with high L/D ratio at large angle of incidence and a new piston analogy has been given for hypersonic flow which can be applied to 2-D aerodynamically optimized body's at large incidence.

\section{ANALysis}

Piston velocity is given by

$U_{P}=U_{1} \sin \alpha+q\left(x-x_{0}\right)$

To co relate the piston velocity with its pressure, Light hill suggested power series expression for pressure on a piston in terms of velocity. The piston velocity should be $\leq$ sound velocity in a free stream.

Pressure ratio is given by

$$
\begin{aligned}
& \frac{P_{2}}{P_{1}}=1+A\left(\frac{U_{P}}{a_{1}}\right)^{2}+A\left(\frac{U_{P}}{a_{1}}\right) \sqrt{B+\left(\frac{U_{P}}{a_{1}}\right)^{2}} \\
& \text { Where, } \quad \mathrm{A}=\frac{\gamma(\gamma+1)}{4} \quad \mathrm{~B}=\left(\frac{4}{\gamma+1}\right)^{2}
\end{aligned}
$$

The nose down restoring moment is given by,

$$
-m=\int_{0}^{L}\left(x-x_{0}\right) P_{2} d x
$$

Where $P_{2}$ stands for windward surface pressure, Leeward surface pressure have not been considered. Aerodynamic stiffness derivative is given by, 
$-c_{m_{\alpha}}=(\gamma+1) \tan \theta\left[2+\frac{\sqrt{M_{1}^{2} \sin ^{2} \theta+\left(\frac{4}{\gamma+1}\right)^{2}}}{M_{1} \sin \theta}+\frac{M_{1} \sin \theta}{\sqrt{M_{1}^{2} \sin ^{2} \theta+\left(\frac{4}{\gamma+1}\right)^{2}}}\right]\left(\frac{1}{2}-h_{0} \cos ^{2} \theta\right)$

Aerodynamic damping derivative is given by,

$$
-c_{m_{q}}=\frac{(\gamma+1) \tan \theta}{\cos ^{2} \theta}\left[2+\frac{\sqrt{M_{1}^{2} \sin ^{2} \theta+\left(\frac{4}{\gamma+1}\right)^{2}}}{M_{1} \sin \theta}+\frac{M_{1} \sin \theta}{\sqrt{M_{1}^{2} \sin ^{2} \theta+\left(\frac{4}{\gamma+1}\right)^{2}}}\right]\left(\frac{1}{3}-h_{0} \cos ^{2} \theta+h_{0}^{2} \cos ^{4} \theta\right)
$$

\section{Results and Discussions}

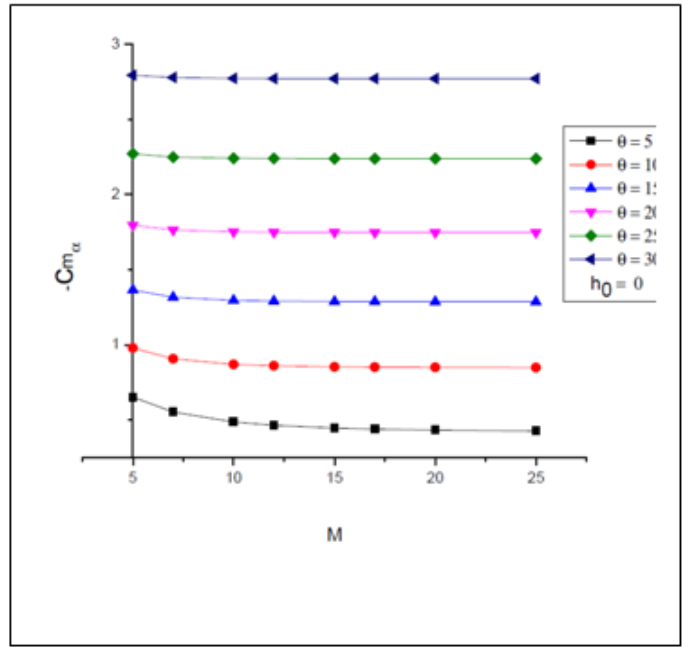

Fig.1 Stiffness Derivative Vs Mach number For $\mathrm{h}_{0}=0$

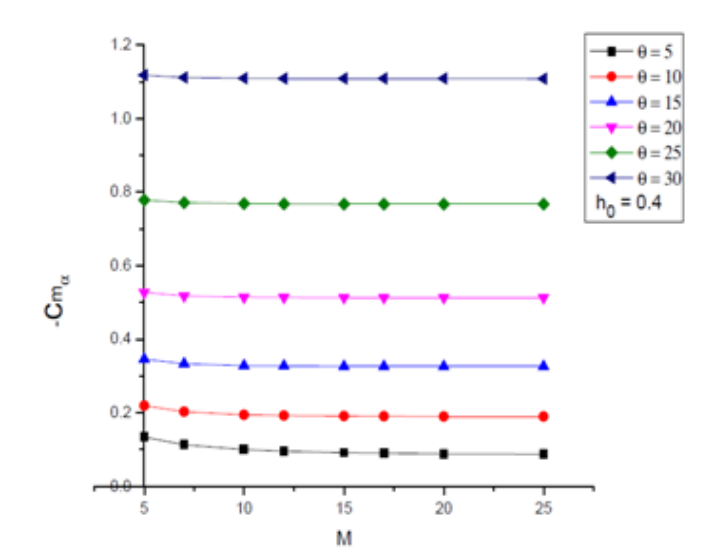

Fig.3 Stiffness derivative Vs Mach number For $\mathrm{h}_{0}=0.4$

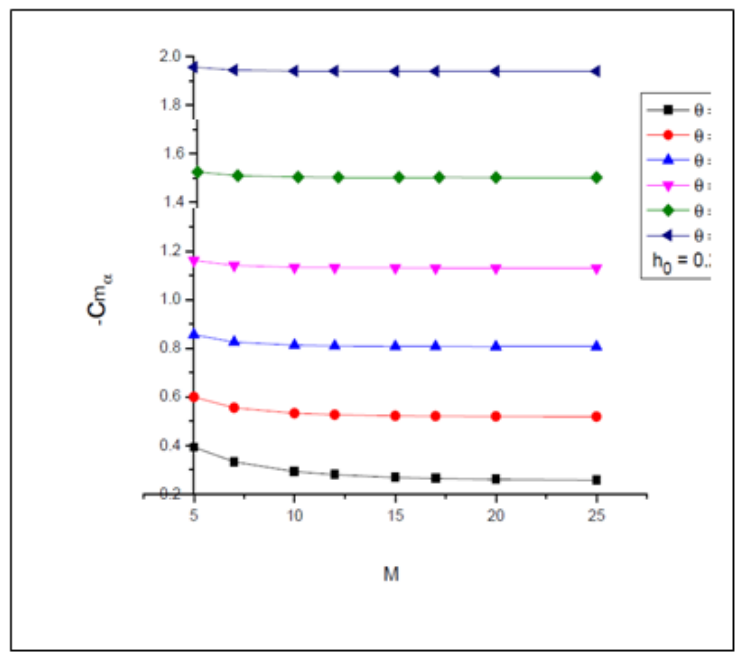

Fig.2 Stiffness Derivative Vs Mach number For $\mathrm{h}_{0}=0.2$

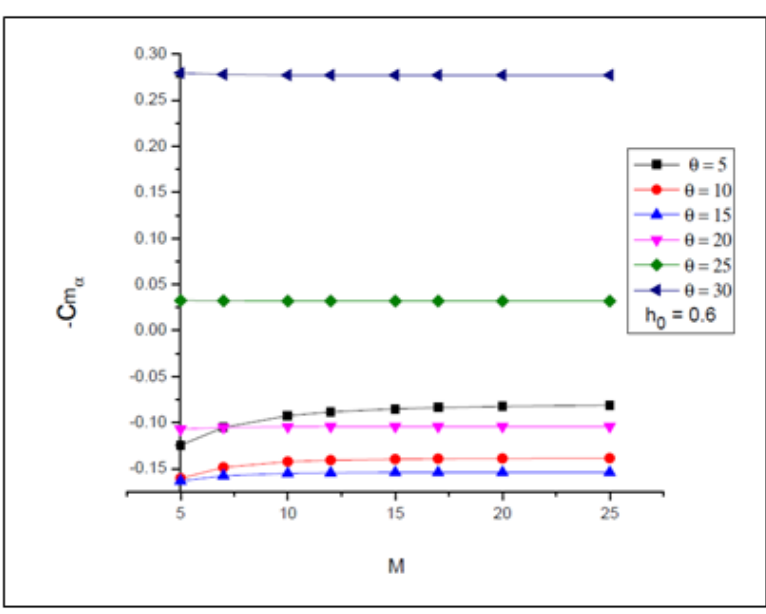

Fig.4 Stiffness derivative Vs Mach number For $\mathrm{h}_{0}=0.6$

Results for pivot position $\mathrm{h}=0$ and 0.2 and 0.4 are shown in Figs. 1 to 3. It is found that for semivertex angle 15 degrees and above the stiffness derivative becomes independent of Mach number and principle of Mach number holds. Whereas for lower semi-vertex angle there is a marginal decrease in the stiffness derivative, this shift is due to the pressure distribution on the surface of the wedge. For Mach number 10 and above the trend is same as discussed above. Fig. 4 shows the results for $\mathrm{h}=0.6$, it is seen that for semi-vertex angle from 5 to 15 degrees and for Mach number 5 to 10 there is marginal increase in the stiffness derivatives and for Mach number $\mathrm{M}=10$ and above it becomes independent of Mach number as seen in figures 1 to 3 . It is also seen that like in figures 1 to 3 where for semi-vertex angles the stiffness derivative was decreased however here for $h=0.6$, increase in inertia level has resulted in increase of stiffness derivative this may be due to the selection of the pivot position which is very close to the center of pressure of the wedge. 
Results for pivot positions $\mathrm{h}=0.8$ and 1.0 are shown in figures 5 and 6 . Figure 5 indicates that for semi-vertex angle theta $=30$ degrees the stiffness derivatives remains constant for the entire range of Mach numbers however, for rest of the values of the semi-vertex angles that the stiffness derivatives increases up to Mach 10 and beyond it becomes independent of Mach number as happened in other cases but for the case of $h=1.0$ refer figure 6 where for all the values of the semi-vertex angles there is non-linear increment in the stiffness derivatives and here also, for Mach number 10 and above the stability derivatives becomes independent of the inertia level and these results are on the expected lines.

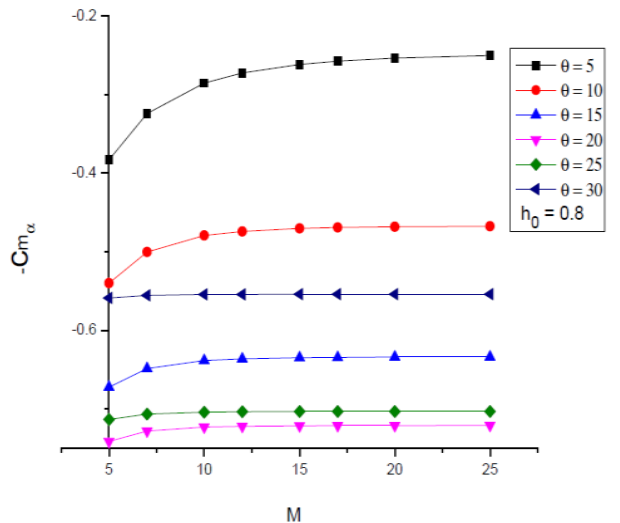

Fig.5 Stiffness derivative Vs Mach number for $\mathrm{h}_{0}=0.8$

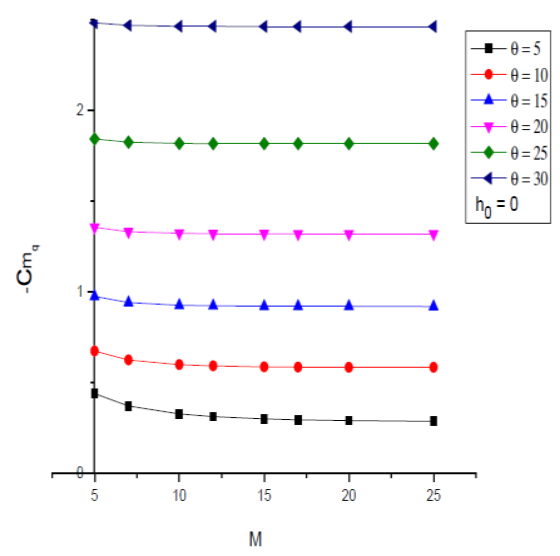

Fig.7 Damping derivative Vs Mach number For $\mathrm{h}_{0}=0$

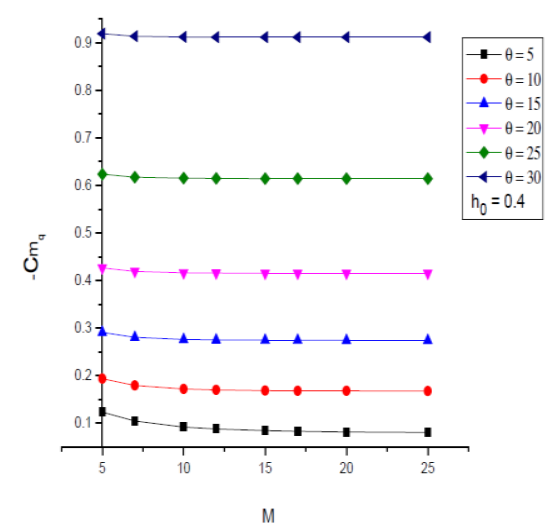

Fig.9 Damping derivative Vs Mach number For $\mathrm{h}_{0}=0.4$

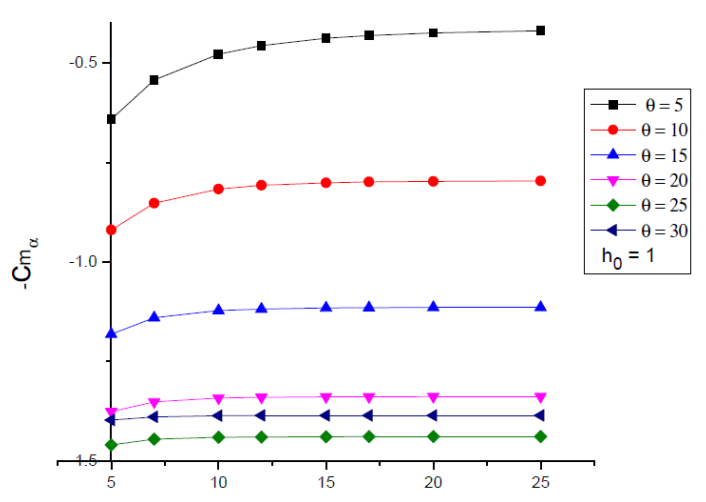

Fig.6 Stiffness derivative Vs Mach number for $\mathrm{h}_{0}=1$

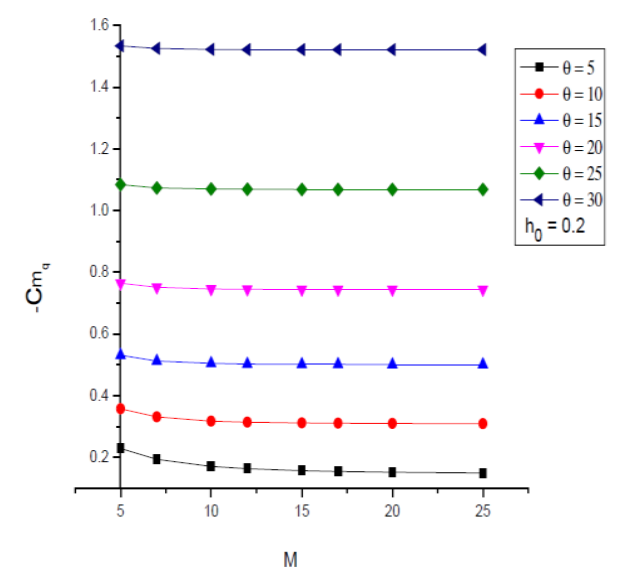

Fig.8 Damping derivative Vs Mach number For $\mathrm{h}_{0}=0.2$

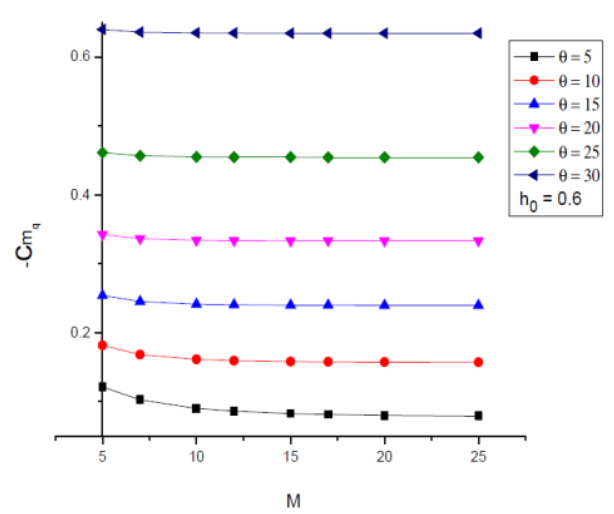

Fig.10 Damping derivative Vs Mach number For $\mathrm{h}_{0}=0.6$ 


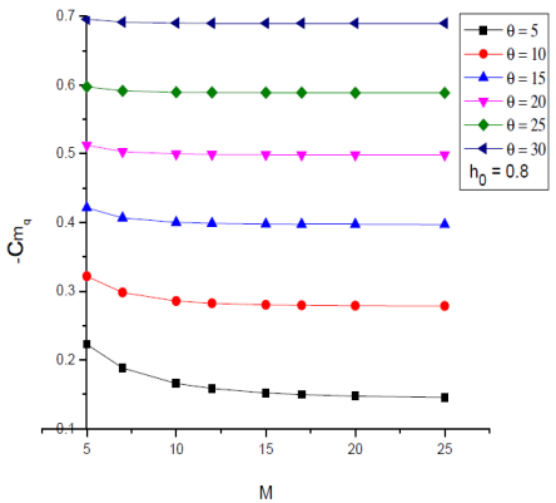

Fig.11 Damping derivative Vs Mach number For $\mathrm{h}_{0}=0.8$

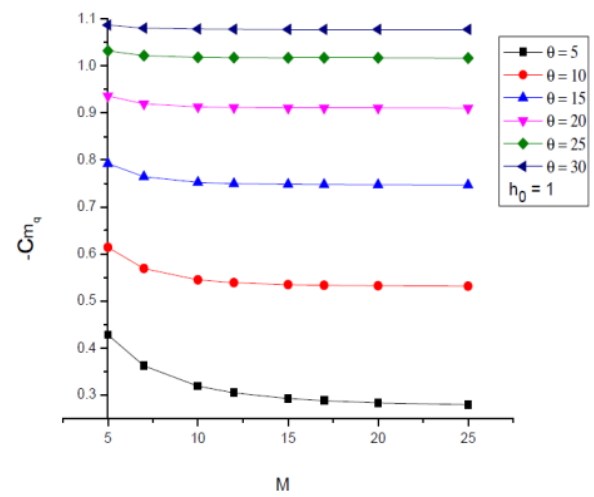

Fig.12 Damping derivative Vs Mach number

For $\mathrm{h}_{0}=1$

Figs 7 to 12 shows the variation of damping derivatives for $h 0=0,0.2,0.4,0.6,0.8$, and 1.0 corresponding to the Mach numbers $M=5$ to 25 for various semi-vertex angle from $\Theta=5$ to 30 degrees. As we have seen that in case of the stiffness derivatives the dependence of the stiffness derivative that for pivot positions $h=0,0.2$, and 0.4 the stability derivatives decreases marginally then achieves the steady state and becomes independent Mach number for $M=10$ and beyond. However, trend reversal takes place for $h=0.6,0.8$ and 1.0. Here in case of damping derivatives the trend is uniform for the entire range of the pivot positions. This uniform behavior is attributed to the non-linear expression of the damping derivative, the location of the pivot position, the pressure distribution on the surface of the wedge, and the variations in the center of pressure due to change in the semi-vertex angles and hence the plan-form area of the wedge. Due to the progressive increase in the plan-form area of the Wedge and also due to the selection of the pivot position it shifts larger area towards the trailing edge leads to such increase in the stability derivatives.

\section{Conclusions}

Based on the above discussions we can draw the following conclusions:

- Stiffness derivatives decreases linearly with the increase in the Mach number for $h=0,0.2,0.4$ but for Mach number 10 and above the steady state is achieved and stability derivative becomes independent of Mach number. For pivot positions $\mathrm{h}=0.6,0.8$, and 1.0 unlike for $\mathrm{h}=0$ to 0.4 the stability derivative increases up to Mach 10 and again it becomes independent of Mach number.

- The variation of the damping derivatives is unaltered for all the values of the pivot positions.

- The damping derivative decreases marginally and then it is independent of Mach number.

- Viscous effects, secondary wave reflections are neglected in the present study.

- In the present study the real gas effects and leading edge bluntness is not taken into considerations.

\section{References}

[1] Tsien, H. S., Similarity laws of hypersonic flow, Journal of Mathematical Physics, 25, 1946, 247-251.

[2] Hayes, W.D. and Probstein, R.F, Viscous Hypersonic similitude, Jr. Aerospace science,.26., 1959, 815-825.

[3] Sychev, V. V, Three Dimensional Hypersonic Gas Flow Past Slender Bodies at High Angles of Attack, Journal of Applied Mathematics and Mechanics, 24, 1960, 296-306.

[4] Lighthill, M. J., Oscillating airfoil at high Mach numbers, Journal of Aeronautical Sciences, 20, 1953, 402-406.

[5] Asha Crasta, M. Ahmed Ali Baig, S. A. Khan , 2012, 'Estimation of Stability derivatives of a Delta wing in Hypersonic flow', International Journal of Emerging trends in Engineering and Developments, Vol. 6, Issue2, Sep., pp. 505-516.

[6] Ghosh K, Mistry B. K., Large incidence hypersonic similitude and oscillating non planer wedges, AIAA J., 18, 1980, 10041007.

[7] Ghosh, K., A new similitude for Aero-foils in Hypersonic Flow, Proceedings of the 6th Canadian congress of Applied Mechanics, Vancouver, Canada, ,1977, 685-686.

[8] Asha Crasta and S. A. Khan, Hypersonic Similitude for Planar Wedges, International Journal of Advanced Research in Engineering and Technology, 5( 2), 2014, 16-31

[9] Asha Crasta and S. A. Khan, Estimation of Stability Derivatives for a Planar Wedge in the Newtonian Limit, IOSR Journal of Mathematics, 10( 2), 2014, 01-06.

[10] Asha Crasta and S. A. Khan, 2014, Effect of Mach number on Stiffness and Damping Derivatives for Oscillating Hypersonic Non-Planar Wedge, IOSR Journal of Mechanical and Civil Engineering, 11( 2), 2014, 04-11.

[11] Asha Crasta and S.A. Khan, Effect of Angle of Incidence on Stiffness and Damping derivatives for Oscillating Hypersonic Non-planar Wedge, International Journal for Research in Applied Science and Engineering Technology,. 2, 2014, 229-242. 